\title{
Stage II Thymic Epithelial Neoplasm AJCC v8
}

National Cancer Institute

\section{Source}

National Cancer Institute. Stage /I Thymic Epithelial Neoplasm A/CC v8. NCI Thesaurus. Code C136323.

Stage II includes: T2, N0, M0. T2: Tumor with direct invasion of the pericardium (either partial or full thickness). N0: No regional lymph node metastasis. M0: No distant metastasis. (AJCC 8th ed.) 